\title{
Is there an elephant in the room?
}

I am a GP in Wales. My pay has been dependent upon my performance, the performance of the nursing and management team of my practice, and of my patients since April 2004. If the patients take my advice and the tablets I prescribe, if our call and recall systems run effectively, and we are careful to put all relevant data into the computer system, then the Quality and Outcomes Framework (QOF) will reward me handsomely.

At the beginning of the QOF payment system, our practice team agreed to enter all blood pressure values taken on the sphygmomanometer into the patient computer records using the recommended codes. We did not wish to be vulnerable to charges of fraud or cheating or to compromise our professional values as doctors and nurses. At the end of the first year of the new payment system, our practice ranked 146th above the lowest achieving practice in Wales for blood pressure target achievement in the diabetes clinical domain. The data for 2007-2008 are now available and we are now ranked as the 25th practice from the bottom of the Welsh league. Our achievement seems poor and seems to be getting worse.

Where are the lines between accuracy, probity, game playing, cheating, and fraud when it comes to making a computer entry of a blood pressure reading? As a practice we decided to draw our line at what we thought was the minimum for ethical, professional practice. Where do you draw the line?
Mrs Jones' systolic blood pressure reading is above the target value. If it is $151 \mathrm{mmHg}$ can I enter 150 into her records with a clear conscience since everyone knows that the machines are not that accurate? If 151 can become 150, what about 152, 153 or 154 ? How many millimetres of mercury matter clinically, how many ethically, and how many financially?

Mr Williams' three readings include one systolic value below the target value and one diastolic value below the target I am aiming for. What is a 'blood pressure reading'? Surely I can enter the belowtarget systolic and the below-target diastolic readings as a record of today's blood pressure, as I am not going to change his medication anyway? His risk of

\section{COMMENTARY}

This essay raises a number of important questions for primary care, none of which have easy answers. ${ }^{1}$ Professor Richards is right to highlight the dangers of a continuum from probity to game playing and perhaps fraud, and to highlight the 'within practice' tensions that can be created when practitioners sit at different places on this spectrum. For the record, I agree with his approach and think the moment you start bending QOF rules and stray from an essentially purist view of blood pressure recording or exception reporting, you enter tiger country. However, I disagree with his assertion around gaming that 'after all everyone is doing it.' The evidence to date, though limited, suggests we are not.

We know from Tim Doran's work on exception reporting that in the second year of QOF, ${ }^{2}$ practices exception reported a median of $5.3 \%$ of patients (interquartile range $=4.0-6.9$ ). There was variation in this generally low level of reporting depending on the type of indicator (higher in the more demanding intermediate outcome than process measures) which could be interpreted as gaming. However, it is far more likely to reflect legitimate clinical concerns. Analysis of Scottish data found that rates of exception reporting in 2005-2006 were higher for practices that had levels of achievement below the maximum thresholds in the previous year than for those that had levels above the maximum thresholds. ${ }^{3}$ Once again it is possible that some practices gamed exceptions to maximise their income. However, the lack of association between the rate of exception reporting and the size of financial incentive attached to each indicator suggests that extensive gaming of exception reporting is unlikely to have occurred. So, while I agree that there is a grey animal in the room, I suspect it's of mouse-like proportions.

There is, however, a caveat. My worries around QOF, motivations, money, and behaviour are far greater for the next generation of doctors than the current ones. Medical students, GPs in waiting, are becoming rapidly accustomed to media descriptions of our avarice and will be initiated, while in debt, into a culture where pay for performance makes up $20 \%$ of their income. Will it be harder to take a purist approach in those circumstances? One solution might be to decrease the financial importance of QOF in line with other international pay-for-performance systems. ${ }^{4}$ I wonder, then, if even the mouse would get out of bed?

\section{Helen Lester}

\section{REFERENCES}

1. Richards J. Is there an elephant in the room? Br J Gen Pract 2009; 59: 376-377.

2. Doran T, Fullwood C, Reeves D, et al. Exclusion of patients from pay-for-performance targets by English physicians. N Engl J Med 2008; 359: $274-284$.

3. Gravelle H, Sutton M, Ma A. Doctor behaviour under a pay for performance contract: further evidence from the Quality and Outcomes Framework. CHE research paper 34

University of York: Centre for Health Economics, 2008.

4. Rosenthal M, Landon B, Howitt K, et al. Climbing up the pay for performance learning curve: where are the early adopters now? Health Affairs 2007 ; 26: 1674-1682. 